\title{
EL CABILDO CATEDRAL CORDOBÉS EN EL PONTIFICADO DEL CARDENAL SALAZAR. CUESTIONES DE MÉTODO Y PRIMERAS APORTACIONES
}

\author{
POR \\ María PAloma de las Mercedes EnríQuez García ${ }^{1}$ \\ Universidad de Córdoba
}

\begin{abstract}
RESUMEN
Como recoge su título, este trabajo pretende reflexionar sobre la forma y el fondo de una fuente importante para estudiar el cardenal Salazar y su cabildo catedral. Esto es, poner de manifiesto las luces y las sombras de las actas capitulares del pontificado de Salazar para estudiar sus relaciones con el cabildo como materia básica a través de la cual conocer la relación entre aquellos dos polos; y mostrar qué personas, procesos y decisiones son los elementos que aquella huella permite revelar y, por ende, el contenido sustancial de esta investigación.
\end{abstract}

PALABRAS CLAVE: obispado; prelado; cardenal Salazar; cabildo catedral; Córdoba; Antiguo Régimen.

\section{CORDOVAN CATHEDRAL CHAPTER DURING THE PONTIFICATE OF CARDINAL SALAZAR. METHODOLOGICAL QUESTIONS AND FIRSTS CONTRIBUTIONS}

\begin{abstract}
As its title shows, this work intends to reflect on the form and the background of an important source to study cardinal Salazar and his cathedral Chapter. That is, to reveal the lights and the shadows of the episcopal capitular acts of that pontificate as the basic matter through which to know the relation between those two poles; and to show what people, processes and decisions are the elements that trace that reveals and, therefore, the substantial content of this research.
\end{abstract}

KEY WORDS: bishopric; prelate; cardinal Salazar; cathedral chapter; Córdoba; Ancient Regime.

CÓMO CITAR ESTE ARTículo / CITATION: Enríquez García, M. P. de las M. 2019. «El cabildo catedral cordobés en el pontificado del cardenal Salazar. Cuestiones de método y primeras aportaciones». Hispania Sacra 71, 143: 249-257. https://doi.org/10.3989/hs.2019.018

\author{
Recibido/Received 27-01-2017 \\ Aceptado/Accepted 09-02-2017
}

La Córdoba con la que el cardenal Salazar se topa a su llegada el miércoles de ceniza de 1687 está, al igual que el resto de España en general y Andalucía en particular, en gran medida imbuida por el Barroco. Aplicando la herencia tridentina, ${ }^{2}$ al protestantismo se le contrarresta con religiosidad en todas sus formas en un momento coincidente con el progresivo declive de la monarquía hispánica por, entre otras razones, las crisis económicas, las acciones de los validos regios y el descontento social, quedando todo ello plasmado en las ingentes producciones culturales del Siglo de Oro. ${ }^{3}$
A esa Córdoba entre el Seiscientos y el Setecientos llegará, en los primeros meses de 1687, como ya se ha dicho, el cardenal Salazar, nacido, como estudia María Soledad Gómez Navarro, en Málaga en 1630 y muerto en Córdoba en 1706. Su padre es regidor y su familia presenta una condición nobiliaria. El eclesiástico es sumamente consciente de sus cargos y atribuciones: mercedario y general de su orden en 1670, predicador real y amigo ${ }^{4}$ de Carlos II, obispo de Salamanca ${ }^{5}$ desde 1681 a 1686, nombrado cardenal por Inocencio XII en 1686 y obispo de

\footnotetext{
1 |22engam@uco.es/ORCIDiD:https://orcid.org/0000-0003-1223-5184

2 Reder Gadow 2000.

3 Domínguez Ortiz 1978.
} 
Córdoba 6 entre 1686 y 1706.

La Córdoba a la que el prelado arriba se caracteriza por un trazado urbanístico prácticamente inalterado desde el comienzo de la época moderna, bache demográfico significativo, economía principalmente agraria, sociedad estamental con acusados contrastes y organización políticoinstitucional y cultural prácticamente incólume, en lo primero, y muy vertida en lo popular en lo segundo.

Con nuestro trabajo pretendemos lograr dos objetivos. EI primero de ellos es poner de manifiesto las luces y las sombras de las actas capitulares de aquel pontificado como materia básica a través de la cual conocer la relación entre aquellos dos polos: obispo y cabildo analizando, comprendiendo y explicando las distintas estrategias metodológicas que tal fuente exige. Entre ellas están la elección del período de gobierno de aquel prelado para ese indicado inicial acercamiento a su figura, el control y el tratamiento de la información que interesa o el imprescindible estado de la cuestión. El segundo es desvelar personas, asuntos y dinámica -o lo que es lo mismo, protagonistas, temáticas y decisiones, procesos, en definitiva- como vectores de esta investigación, concebida como una aportación a la Historia Social y de las Instituciones a través de la Historia de la Iglesia de la España del Antiguo Régimen. El enfoque de nuestra labor es local -que no localista- por el ámbito y proyección de los cabildos catedralicios, por lo que realmente nos proponemos presentar un modelo metodológico y explicativo aplicable a otros casos nacionales. Por estos ingredientes, se convierte en verdadera contribución al conocimiento y a la historiografía modernista.

El desarrollo de ambos fines constituye, pues, las dos partes de este texto, al que abrocharán las consideraciones que al respecto se puedan derivar o desgajar.

\section{UNA HUELLA IMPORTANTE PARA CONOCER LOS CABILDOS CATE- DRALICIOS EN LA ESPAÑA MODERNA: LAS ACTAS CAPITULARES COMO CONTINENTE Y CONTENIDO}

En este artículo damos a conocer un paso previo a la completa redacción de la biografía del cardenal Salazar. Dicha biografía se materializa en una interesante e inexplorada parcela alusiva a las relaciones cardenal-cabildo durante tres de los veinte años que compendian su episcopado en la ciudad cordobesa (1686-1706). Es imprescindible conocer ciertos elementos del cabildo catedralicio cordobés dentro de una variable espacio-temporal concreta, a caballo entre las dos décadas finales del siglo XVII y la primera del siguiente. Tales matices son: definición, composición, características, funciones, intervinientes, asuntos a dirimir, evolución a lo largo del tiempo, influencia en su ciudad y su entorno y estado de sus relaciones con las restantes instituciones eclesiásticas, civiles, reales, y, por supuesto,

$6 \quad$ Nuestro trabajo parte de una línea de investigación propia más amplia dirigida a elaborar la biografía del cardenal Salazar. Este trabajo representa una ampliación muy considerable del que debimos defender en forma de póster en la Universidad de Córdoba como actividad obligatoria en nuestra condición de becaria de sendas becas de iniciación a la investigación y de colaboración. Primer peldaño hacia nuestra meta final, y avance de lo que ya empezamos a saber sobre el cardenal Salazar y su cabildo catedral, pretende ahora fijarse en forma y fondo, como recoge su título y se ha indicado en el resumen. con la sociedad cordobesa del momento.

Por tanto, las directrices fundamentales de esta investigación se compendian en tres: analizar la Córdoba y la persona del cardenal Salazar como marcos indispensables de contexto para su actuación como prelado, estudiar el cabildo catedralicio cordobés como institución e imprescindible contexto institucional a la labor de Salazar, y examinar y explicar el cabildo catedralicio cordobés a través de la sociedad que pasa por él y sus preocupaciones durante tres años del obispado del purpurado, como son los correspondientes a sus inicios (16861687); su mitad (1696-1697) y su final (1705-1706).

Nuestra investigación se cimenta en el estudio de las inéditas actas capitulares que por espacio de dichos años el cabildo catedralicio cordobés produce durante su episcopado. Conservadas en el Archivo Capitular de la Catedral de Córdoba, constituyen un pilar nuclear de la investigación en curso amén de ser el único fehaciente vestigio de la actividad episcopal del obispo-cardenal Salazar y de sus líneas de actuación en su ente capitular. Se analizan como continente y como contenido para conocer los asuntos y cuestiones que plantea y que aquí se expondrán.

Del tema que estamos presentando, pese a su inicial carácter local, no puede perderse de vista su dimensión nacional, en tanto que Córdoba es paradigma de cabildo y obispado en la época que estamos historiando -es el destino soñado por todo eclesiástico por las pingües rentas que ofrece, por su amplia capacidad de acción y por sus buenas relaciones tanto con la Corona, como con los restantes cabildos diocesanos españoles, de entre los que sobresale el arzobispado de Toledo, del que, al igual que los otros, depende,- 7 y ha sido estudiado por un acreditado grupo de especialistas en la Iglesia moderna española de la talla de Álvarez Santaló, Barrio Gozalo, Domínguez Ortiz, García-Cuevas Ventura, Jiménez Martínez, Morgado García o Vázquez Lesmes.

Todos ellos tocan aspectos que nos han resultado interesantes para nuestra investigación. A Álvarez Santaló se le deben importantes avances en psicología y modus vivendi del eclesiástico en el Antiguo Régimen, ${ }^{8}$ sobresalientes son las aportaciones de Barrio Gozalo ${ }^{9}$ y de Vázquez Lesmes ${ }^{10}$ sobre el clero español moderno partiendo del modelo cordobés, magistral es el enfoque que Domínguez Ortiz hace de la Iglesia española del Antiguo Régimen como élite privilegiada y de poder desde la perspectiva de la Historia social, ${ }^{11}$ muy de notar las averiguaciones de Martínez Jiménez sobre las relaciones obispo-capitulares en la diócesis malagueña en tiempos de los primeros Borbones y en un momento de significativas modificaciones en el horizonte eclesiástico ${ }^{12}$ e imprescindible la contextualización que nos hace García-Cuevas Ventura sobre la institución capitular catedralicia cordobesa en el abanico cronológico que va desde la Revolución francesa hasta la Restauración. ${ }^{13}$

Cuerpos colegiados caracterizados por ser los principales ejes vertebradores del gobierno diocesano y por consti-

\footnotetext{
7 Vázquez Lesmes 1987: 145-147.

8 Álvarez Santaló 2010.

9 Barrio Gozalo 2010: 29-34.

10 Vázquez Lesmes 1987: 239-244.

11 Domínguez Ortiz 1985: 215-247.

12 Véase Jiménez Martínez 2007.

13 García-Cuevas Ventura 1996: 99.
} 
tuir los idóneos ámbitos para el desarrollo de un doble juego de fuerzas a tenor de la coyuntura histórica y la relación prelado-capitulares y viceversa, los cabildos catedralicios se convierten en piezas fundamentales de las organizaciones e instituciones eclesiásticas de la España Moderna. ${ }^{14}$ Esta relación está determinada por intereses y conveniencias internas - casa, comida y vestido- y externas - problemas a los que hacer frente-, y es el condicionante del funcionamiento y capacidad de resolución de este órgano de los problemas de la sociedad que pasa por él.

Lo anterior se cumple en el cabildo catedralicio cordobés, nacido en 1237 por la conquista de Córdoba un año antes por Fernando III el Santo, en el período transicional del siglo $\mathrm{XVII}$ al XVIII, con unos protagonistas concretos: el cardenal Salazar y su cabildo catedral. ${ }^{15}$

Su estructura es piramidal. En la cúspide está el obispo y conforme se desciende a la base se encuentran, por este orden, canónigos, racioneros o porcioneros, deanes, arcedianos, chantres y tesoreros. Esta disposición jerárquica aparece con una cierta simultaneidad pareja a organización y desarrollo del ente capitular ${ }^{16}$. De acuerdo con los estatutos del obispo Fresneda, y siguiendo las tesis de Aranda Doncel, el cabildo catedral cordobés se integra por ocho dignidades: deán, arcediano de Córdoba, maestrescuela, arcediano de Castro, chantre, arcediano de Pedroche, tesorero y prior. ${ }^{17}$

Sus características varían dependiendo del cabildo al que nos estemos refiriendo, por motivos directamente relacionados con solvencia económica y situación geográfica. En virtud de su ubicación, del favor real y de su capacidad financiera, el cabildo tendrá más prestigio y prebendas. No es lo mismo el arruinado e insignificante cabildo de Ciudad Rodrigo que el boyante cabildo cordobés, con unas rentas en su haber nada despreciables y un descomunal engrosado patrimonial financiado por dos vías: directa e indirecta. ${ }^{18}$

La vía directa es la relativa a los ingresos obtenidos por las obras pías, donaciones de particulares en tierras y/o dinero y donaciones reales. Estas donaciones directas son el grueso patrimonial físico del cabildo y se complementan con las indirectas, que, entre otras, incluyen la concesión de indulgencias papales y de prerrogativas reales prestigiadoras aún más del cabildo beneficiado por ellas. En el caso del cabildo catedralicio cordobés, la riqueza de la mesa capitular se debe a numerosas propiedades rústicas y urbanas, en la percepción de voluminosas rentas por diferentes conceptos con notables disparidades en el valor económico de las prestameras anejas a dignidades y canonjías - motivo de pesquisas que en más de una ocasión aparecen reflejadas en las actas capitulares manejadas para la elaboración de este trabajo-.${ }^{19}$

Sus competencias gubernativas abarcan los ámbitos interno -decretos capitulares, esto es, acuerdos, deliberaciones y votaciones- y externo -asistencia y solvencia de los retos que se le plantean-. Lo anterior se regula por una correcta administración concertada en diferenciados aspectos

\footnotetext{
14 García-Cuevas Ventura 1996: 29-35

15 Aranda Doncel 1984: 48-49.

16 Vázquez Lesmes 1987: 145-157.

7 Aranda Doncel 1984: 48.

Cuenca Toribio 2005:12-20.

19 Aranda Doncel 1984: 49.
}

individuales y colectivos. Diezmos, arrendamientos y pagos son los primeros. Repartos de alcabalas y diezmos y prestameras, los segundos. ${ }^{20}$

Los cometidos de los capitulares se reparten en lectoral, penitenciario, magistral y doctoral, aumentando el esquema conforme abarque más competencias regulándose por estatutos, como, centrándonos nuevamente en Córdoba, los del obispo Fresneda, que, entre otras cosas, dictaminan que sea el obispo el que determine esta cuestión mediante las bulas de nombramiento, la recepción y juramento de estatutos y la resolución, en la medida de lo posible, de este asunto de cara al interregno de la sede vacante. Es, por tanto, en este trascendental documento donde se recogen aspectos de ampliación del espectro capitular, económicos y de política exterior, componiendo este último marco las relaciones tanto con los monarcas como con entidades pías, gremiales y cofrades. También se indican los requisitos a cumplimentar para ingresar en el cabildo -acreditación de estudios y limpieza de sangre-, los derechos de sus miembros y las funciones a desempeñar en función de su rango. ${ }^{21}$

Aunque estas premisas parezcan expuestas tan rígidamente, serán violadas por algunos prelados como Salazar, quien aplicará excepciones con sus afines y familiares.

En todo el contexto indicado, la aportación de este trabajo y su contenido a la historiografía eclesiástica es conocer el funcionamiento real por dentro y por fuera de un cabildo catedralicio -que es lo que prácticamente brindan las actas capitulares-, cómo es su día a día, qué sociedad -cordobesa en este caso-, qué inquietudes y con qué ritmos pasan por sus manos institucionales, qué problemas dirime.

Aunque muy formal por razones obvias, es indudable que las actas capitulares episcopales como fuente ayudan en gran medida a colmatar y responder todas esas incógnitas interrogándolas bien y adecuadamente; como ya se apuntó, es lo que importa en este apartado, es decir, analizar aquella huella documental en su aspecto formal como fuente, con sus luces y sus sombras así como en las decisiones metodológicas que su examen exige e implica.

Si bien la metodología de esta investigación descansa en los consabidos pasos indispensables de toda investigación, nos centramos en las fuentes primarias, por lo que ahora diremos.

Como en epígrafes anteriores hemos indicado, la fuente de la que manan en su inmensa mayoría información y datos constatables en este trabajo, son las actas capitulares que, por espacio de tres de los veinte años en los que el cardenal Salazar fue obispo de Córdoba, genera el cabildo catedralicio cordobés viéndose en ellas aspectos tan inéditos como trascendentales cuáles son intervinientes, asuntos y dinámica que jalonan la tónica del cabildo catedralicio cordobés dentro de ese tiempo.

Estas actas se conservan en el Archivo Capitular de la catedral de Córdoba (en lo sucesivo expresado como ACCO), y son un vivo testimonio tanto de la labor episcopal de Salazar como de las inquietudes que cierto sector de la sociedad cordobesa lleva a su cabildo con vistas a que este se las resuelva.

\footnotetext{
20 Barrio Gozalo 2010: 175-209.

21 Morgado García 2000: 54-59.
} 
Fotografiadas durante el verano de 2014 y estudiadas hasta mayo de 2016, mi elección recayó en ellas por, aparte de lo ya expresado, explicar de primera mano idiosincrasia y costumbres de Iglesia y sociedad cordobesas como imprescindible elemento de comprensión de una no menos crucial faceta de la Historia de la Iglesia en el Antiguo Régimen; $y$, diseccionadas desde una perspectiva global, vemos que es mayor el número de cabildos plenos ordinarios - doscientos cuarenta y seis - que de cabildos plenos extraordinarios - ciento veinticuatro- y que su estructura se articula en los siguientes elementos:

1.- Una introducción, donde se dice si la reunión celebrada es ordinaria o extraordinaria, junto con fecha y lugar de su desempeño - por lo general en la capilla de san Clemente de la catedral cordobesay total de capitulares asistentes. También se hace notar la presencia o ausencia del obispo - esto último mediante la expresión ya sea en latín ya sea en castellano, de que la sede episcopal está vacante; lo que es una constante hasta el miércoles de ceniza de 1687, día en que Salazar, procedente de Roma, toma posesión de su nueva mitra, hecho que no está exento de polémica-.

2.- Una parte central, correspondiente a un cuerpo textual, en el que tras la frecuente concesión de limosna, se expone el asunto convocatorio de la sesión.

3.- Una parte final en la que, en función de las circunstancias, se resuelve o se demora el problema planteado - lo que es poco habitual, dada la pronta disposición capitular pese a las cortapisas infligidas por su prelado-.

Si el conflicto se resuelve, así se manifiesta en el acta poniéndose seguidamente la solución dada; y de demorarse el asunto, el cabildo crea una comisión que valora el caso que se le propone y dictamina un veredicto tras un indefinido tiempo de deliberación - de un par de semanas a varios años-. No obstante, debemos tener presente que la documentación estudiada de dos décadas abarca solo tres años, con la lógica pérdida de rastro de multiplicidad de asuntos y temas a tratar.

Las actas se cierran con la firma del secretario o secretarios capitulares de turno. Para el caso del primer año, esto es, 1686-1687, los secretarios son: don Diego Mesía de la Cerda, don Diego de Bañuelos, don Francisco de Llanes y Cárdenas y el arcediano de los Pedroches. Para 1696-1697: don Francisco de Eraso y Frío, don Luis Beltrán de Guevara, don Diego de Bañuelos, don Alonso Gómez de Cárdenas y don Alonso Fausto de Valderrama. Y para 1705-1706 le toca el turno a don Francisco de Valenzuela y Cañete, don Felipe José Morillo Velarde y don Pedro Alfonso Deza y Atienza.

Las trescientas setenta reuniones capitulares que se celebran a lo largo de los aludidos años se acometen con relativa frecuencia, observándose un mayor número de ellas entre 1696 y 1697, después entre 1705 y 1706 y por último entre 1686 y 1687.

Centrándonos de nuevo en el estudio de las actas capitulares, diremos que el método de análisis seguido debe comprender una doble perspectiva: cuantitativa y cualitativa; y ha consistido en búsqueda, recogida e interpretación de las mismas cotejando su contenido con lo que en bibliografía especializada se puede hallar sobre el tema que nos ocupa.

El haber elegido tres años de los veinte en los que en total Salazar fue obispo de Córdoba no es casual, sino que obedece a unos criterios de acotación cronológica que tienen el propósito de acercarnos al episcopado del cardenal Salazar y al funcionamiento de su cabildo en momentos claves de su actividad, como son los correspondientes a sus años de inicio -1686-1687-; su mitad -1696-1697- y su término -1705-1706-.

\section{EL CABILDO CATEDRALICIO CORDOBÉS EN TIEMPOS DEL CARDE- NAL SALAZAR SEGÚN SUS ACTAS CAPITULARES}

En esta sección desvelaremos quiénes pasan por el cabildo catedralicio cordobés, cuáles son los asuntos que este trata y qué posibles cambios se producen y quienes actuarán en estos ámbitos de forma patente. Responderemos estas cuestiones, dentro del señalado abanico cronológico, atentos tanto a las personas físicas -individuos de los distintos estamentos sociales- como a las jurídicas -instituciones- que intervienen.

Comenzando por las personas físicas, como podemos ver en la Tabla 1, diremos que del total de individuos que tenemos, la mayoría son eclesiásticos. Los que más asiduamente intervienen en este organismo son afines a Salazar, como su secretario, el canónigo don Bernardo Blázquez ${ }^{22}$ o su sobrino, don Pedro de Salazar y Góngora ${ }^{23}$. Este último cobra un inusitado protagonismo entre 1705 y 1706 a tenor de la enfermedad y muerte de su tío, a quien sustituirá en la mitra. Otros eclesiásticos muy presentes en la documentación son los miembros de renombradas familias cordobesas como los Góngora, los Argote, los Mesía o los Valenzuela. Su presencia se explica dentro del estamento eclesiástico por la puesta en práctica de una estrategia de promoción familiar dentro del complejo sistema social imperante en la Edad Moderna. ${ }^{24}$

TABLA 1

Relación global de las personas físicas que intervienen y que son mencionadas en las actas del cabildo catedral de Córdoba durante los tres años testigos

\begin{tabular}{|c|c|c|}
\hline & & $\begin{array}{c}\text { TOTAL GLOBAL DE PERSONAS } \\
\text { FÍSICAS } \\
326(100 \%)\end{array}$ \\
\hline & & № / \% \\
\hline \multirow{4}{*}{ ESTAMENTOS } & Eclesiástico & 249 / 76,61 \\
\hline & $\begin{array}{l}\text { Tercer } \\
\text { estado }\end{array}$ & 63 / 19,38 \\
\hline & Nobiliario & $10 / 3,07$ \\
\hline & Realeza & $3 / 0,92$ \\
\hline
\end{tabular}

Fuente: Elaboración propia.

22 Archivo Capitular de la Catedral de Córdoba (en lo sucesivo ACCO), t. 66, f. 315 v.

${ }_{23}$ ACCO, t. 66, f. 330 r.

24 Soria Mesa 2000: 14. 
El segundo grupo con mayor representatividad es el tercer estado, contenido en las actas capitulares en sesenta y tres individuos. Pese su pertenencia en su inmensa mayoría al sector terciario, se reparten en dos miembros del sector primario - un pastor y un agricultor-, tres del secundario -dos molineros y un obrero- y veintiuno del terciario - seis abogados, dos administradores, dos bachilleres, dos arrendadores de diezmos, un barbero, un notario de cruzada, un oficial de cabezas de renta, un procurador y oidor de la Real Chancillería de Granada, un consejero real de Castilla, un corregidor y un ministro-. A esto hay que añadir treinta y siete inclasificables, personas de las que solo se saben su nombre y, en todo caso, si son o no vecinos de Córdoba. De ellos: veintisiete llevan el distintivo «don» frente a los diez restantes que no lo llevan, lo que podría ser indicativo de jerarquizaciones y distinciones que existen y proliferan en el seno de este estamento.

La presencia del sector terciario dentro de las actas capitulares hunde sus raíces, según Aranda Doncel, no en una desaparición de los otros sectores - principales motores económicos del momento- pero sí en un progresivo y paulatino repunte de este sector apreciable entre finales del siglo XVII y principios del siguiente como prueba de las aspiraciones cada vez más altas de las personas a él adscritas, con su consiguiente reflejo en la documentación analizada, yendo todo esto parejo a crecientes procesos de urbanización y alfabetización social. ${ }^{25}$

El estamento nobiliario está representado en los documentos en diez nobles, miembros, a su vez, de muy diversos escalafones dentro del mismo, puesto que tenemos a seis marqueses, un duque, dos caballeros veinticuatros y una condesa, que, en palabras de Soria Mesa, conforman la llamada «nobleza moderna», que ha alcanzado su posición habida cuenta de prestar importantes favores a la Corona y por haberse impuesto al resto del vulgo mediante diversos mecanismos, sobre todo, el cultural. ${ }^{26}$

La realeza es mencionada en las actas en tres miembros - Carlos II, ${ }^{27}$ Felipe $\mathrm{V}^{28}$ y su primera esposa, María Luisa Gabriela de Saboya- ${ }^{29}$ en concordancia con la propia vida de Salazar, en tanto que su fallecimiento se produce en agosto de 1706, en pleno desarrollo de la guerra de sucesión española (1700-1715). Los motivos por los que la realeza aparece en la documentación son: rogativas pro salute en caso del último Austria y motivos derivados del conflicto sucesorio en caso del primer Borbón español y su primera esposa.

En lo tocante a las personas jurídicas, esto es, las instituciones, diremos, como puede verse en la Tabla 2 que las que tienen mayor presencia dentro del ente capitular son los cabildos diocesanos con los que Córdoba interactúa por motivos como las gracias de subsidio y excusado, tema que se prolonga muy repetidamente a lo largo del período estudiado. Entre otros, los cabildos que más destacan son, por este orden, los de Granada, ${ }^{30}$ Málaga, ${ }^{31}$ Sevilla y
TABLA 2

Relación global de las personas jurídicas (instituciones) mencionadas en las actas del cabildo catedral de Córdoba durante los tres años testigos

\begin{tabular}{|l|r|c|c|c|c|}
\cline { 2 - 6 } \multicolumn{1}{c|}{} & \multicolumn{1}{|c|}{$\begin{array}{l}\text { TOTALES } \\
\text { (№ } / \%)\end{array}$} & \multicolumn{4}{c|}{ TOTAL GLOBAL DE INSTITUCIONES } \\
\hline $\begin{array}{l}\text { INSTITUCIONES } \\
\text { ECLESIÁSTICAS }\end{array}$ & $22 / 100$ & $\begin{array}{c}\text { Cabildos } \\
\text { Catedralicios }\end{array}$ & Santa Sede & Colegios & Hospitales \\
\hline $\begin{array}{l}\text { INSTITUCIONES } \\
\text { LAICAS }\end{array}$ & $5 / 100$ & $19 / 86,36$ & $1 / 4,55$ & $1 / 4,55$ & $1 / 4,55$ \\
\hline
\end{tabular}

Fuente: Elaboración propia.

Toledo. ${ }^{32}$ También se contemplan relaciones a gran escala con cabildos catedralicios ultramarinos como el mexicano, la Santa Sede o instituciones como el colegio de san Antonio ${ }^{33}$ o el hospital de niños expósitos. ${ }^{34}$

Las instituciones laicas son, como decimos, cinco y las que tienen una mayor presencia son los concejos municipales, como los de Córdoba ${ }^{35}$ o Puebla de Alférez, ${ }^{36}$ que acuden al cabildo por rogativas pidiendo la lluvia - dada una acuciante sequía, siendo esta una muy común práctica, como estudia Peña Díaz para la Andalucía del Siglo de Oro- ${ }^{37}$ o por impago de deudas.

En conclusión podemos afirmar que registro y representatividad de instituciones en las actas capitulares cordobesas es perfectamente extrapolable al resto de los cabildos diocesanos españoles, ${ }^{38}$ o en su defecto, a los más pudientes, como el granadino, el malagueño, el sevillano o el toledano.

La Tabla 3 nos muestra los asuntos que jalonan la tónica del cabildo catedralicio cordobés durante los aludidos tres años testigos. Su tipología es muy diversa y se reparten de manera relativamente uniforme en las actas, si bien son más numerosos los asuntos internos del cabildo

En una importante pero menor medida el cabildo dirime cuestiones relativas a relaciones entre cabildos, con la Santa Sede y Ultramar y a relaciones Iglesia-Estado, lo que confirma el enorme poder del cabildo catedralicio cordobés y el estrecho vínculo existente en este momento entre el altar y el trono. Otrosí, el cabildo resuelve asuntos que tienen que ver con pleitos y asistencia al clero diocesano, temáticas que aumentan discretamente con el paso de los años.

Todo lo anterior está jalonado por las relaciones entre el cardenal Salazar y su cabildo catedral, contenidas en treinta ocasiones en las actas capitulares, y que parten de este axioma: un constante e intenso juego de fuerzas entre el prelado y sus subordinados por la hegemonía

\footnotetext{
25 Aranda Doncel 1984: 19-35.

26 Soria Mesa 2000: 80.

27 ACCO, t. 62, f. 346 r.

28 ACCO, t. 66, f. 346 r.

29 ACCO, t. 66, f. 266 r.

30 ACCO, t. 62, f. 103 v.

31 ACCO, t. 64 , f. 438 v.
}

$\begin{array}{ll}32 & \text { ACCO, t. 66, f. } 294 \text { v. } \\ 33 & \text { ACCO, t. 62, f. } 96 \text { v. } \\ 34 & \text { ACCO, t. 62, f. } 368 \text { r. } \\ 35 & \text { ACCO, t. 66, f. } 243 \text { r. } \\ 36 & \text { ACCO, t. 66, f. } 255 \text { v. } \\ 37 & \text { Peña Díaz 2014: } 7 . \\ 38 & \text { Pons Alós y Cárcel Ortí 2013: 99-101. }\end{array}$ 
TABLA 3

Relación global de los asuntos tratados por el cabildo catedral cordobés durante los tres años testigos

\begin{tabular}{|l|l|l|l|l|l|l|l|}
\hline \multicolumn{7}{|c|}{ TOTAL: 283 (100\%) } \\
\hline $\begin{array}{l}\text { Personal del } \\
\text { cabildo } \\
\text { (№ / \%) }\end{array}$ & $\begin{array}{l}\text { Asuntos } \\
\text { económicos } \\
\text { (№ / \%) }\end{array}$ & $\begin{array}{l}\text { Relaciones } \\
\text { cardenal- } \\
\text { cabildo } \\
\text { (№ / \%) }\end{array}$ & $\begin{array}{l}\text { Relaciones } \\
\text { entre cabildos la } \\
\text { Santa Sede y/o } \\
\text { Ultramar } \\
\text { (№ / \%) }\end{array}$ & $\begin{array}{l}\text { Relaciones } \\
\text { Iglesia- } \\
\text { Estado } \\
\text { (№ / \%) }\end{array}$ & $\begin{array}{l}\text { Asistencia a la } \\
\text { sociedad (№ } \\
\text { /\%) }\end{array}$ & $\begin{array}{l}\text { Pleitos } \\
\text { (№ / \%) }\end{array}$ & $\begin{array}{l}\text { Asistencia al } \\
\text { clero diocesano } \\
\text { (№ / \%) }\end{array}$ \\
\hline $146 / 51,59$ & $43 / 15,19$ & $30 / 10,60$ & $23 / 8,13$ & $16 / 5,65$ & $14 / 4,95$ & $7 / 2,47$ & $4 / 1,41$ \\
\hline
\end{tabular}

Fuente: Elaboración propia.

capitular. Por una parte, Salazar, muy observante de la legislación tridentina, intervendrá muy virulentamente en el cabildo para reafirmar su autoridad. Por otra, los capitulares responderán a las exigencias de su prelado desde su colegialidad reivindicando, siempre que pueden, su idiosincrasia y autonomía.

Es una verdadera obsesión de Salazar, tal como demuestran los documentos de los cabildos plenos ordinarios del 14 de febrero de $1687^{39}$ y del 13 de agosto de $1697,{ }^{40}$ que se le presenten con gran regularidad las cuentas de su obispado, - ya sea de sus propiedades, de los vecinos que le adeudan dinero o de los sueldos de sus capitulares - como medio de imponerse sobre el ente capitular y nombrará y destituirá a dedo dentro de dicho organismo, tejiendo en él una red clientelar a la que colocará en estratégicos puestos que le otorgarán su tan ansiada dominancia.

También serán famosas las tiranteces por cuestiones protocolarias, y prueba de ello es la sesión capitular del miércoles de ceniza de 1687 - cuando, con motivo de la toma de posesión de su mitra en Córdoba, el purpurado exige se le coloque desde entonces y para siempre un dosel color púrpura en el púlpito de la catedral cordobesa cada vez que predique allí, para extrañeza de sus capitulares,${ }^{41}$ sus interferencias en asuntos de exclusiva competencia capitular como es la gestión de los pleitos - sesiones capitulares extraordinarias del 28 de febrero ${ }^{42}$ y del 14 de mayo de $1697^{43}$ y cabildo pleno ordinario del 22 de mayo de $1697-, 44$ sus imposiciones a sus subordinados como el cumplimiento de las disposiciones testamentarias de sus albaceas ${ }^{45}$ y la asiduidad con la que visita su obispado acompañado de personas que le son leales como, por ejemplo, el canónigo don Juan Antonio de Victoria. ${ }^{46}$

Vistos personas, instituciones y problemática que jalonan la tónica capitular cordobesa, en este momento, atraeremos nuestra atención en la evolución cronológica de los mismos durante los tres años testigos, observando si hay o no continuidad en ellos.

La Tabla 4 nos muestra la evolución en los casos de las personas físicas y jurídicas -instituciones- que acuden al

\footnotetext{
39 ACCO, t. 62, f. 160 v.

40 ACCO, t. 64, f. 461 v.

41 ACCO, t. 62, f. 405 r.

42 ACCO, t. 62, f. 179 v.

43 ACCO, t. 64, f. 425 v.

44 ACCO, t. 64, f. 431 r.

45 ACCO, t. 64, f. 405 r.

46 ACCO, t. 64, f. 416 v.
}

TABLA 4

Evolución cronológica de los intervinientes que durante los tres años testigos Ilevan sus problemas al cabildo catedralicio cordobés

\begin{tabular}{|l|l|c|c|c|c|}
\hline \multicolumn{2}{|l|}{} & $\begin{array}{l}\text { TOTALES } \\
\text { (№ } / \%)\end{array}$ & $1686-1687$ & $1696-1697$ & $1705-1706$ \\
\hline \multirow{4}{*}{$\begin{array}{l}\text { PERSONAS } \\
\text { FÍSICAS }\end{array}$} & $\begin{array}{l}\text { Estamento } \\
\text { eclesiástico }\end{array}$ & $249 / 100$ & $58 / 23,29$ & $95 / 38,15$ & $96 / 38,55$ \\
\cline { 2 - 7 } & $\begin{array}{l}\text { Tercer } \\
\text { estado }\end{array}$ & $63 / 100$ & $13 / 20,63$ & $21 / 33,33$ & $29 / 46,03$ \\
\cline { 2 - 7 } & $\begin{array}{l}\text { Estamento } \\
\text { nobiliario }\end{array}$ & $10 / 100$ & $3 / 30$ & $1 / 10$ & $6 / 60$ \\
\cline { 2 - 7 } & Realeza & $4 / 100$ & $1 / 25$ & $1 / 25$ & $2 / 50$ \\
\hline \multirow{2}{*}{$\begin{array}{l}\text { PERSONAS } \\
\text { JURÍDICAS } \\
\text { (INSTITUCIONES) }\end{array}$} & Eclesiásticas & $22 / 100$ & $4 / 18,18$ & $12 / 54,55$ & $6 / 27,27$ \\
\cline { 2 - 7 } & Laicas & $5 / 100$ & $1 / 20$ & - & $4 / 80$ \\
\hline
\end{tabular}

Fuente: Elaboración propia.

cabildo catedral cordobés durante el señalado período de la prelatura de Salazar.

Convendría, antes de entrar en materia, mencionar que percibimos más continuidad en la evolución de los intervinientes físicos y jurídicos del estamento eclesiástico frente a los demás.

Concentrándonos ya en el cuadro, examinaremos sucintamente la trayectoria de las personas físicas. Si a lo largo de los tres años testigos son doscientos cuarenta y nueve los eclesiásticos que actúan y participan en el cabildo de la catedral de Córdoba, ese total se reparte en cincuenta y ocho para 1686-1687, noventa y cinco para 1696-1697 y noventa y seis para 1705-1706. Dentro de este grupo de los que se puede establecer un más palpable seguimiento son, aparte del cardenal Salazar - protagonista de nuestro trabajo, y cuya intervención activa consta en ocho ocasiones para el primer año testigo, en doce para el segundo y en dos para el tercero- los diversos afines al cardenal Salazar; en definitiva, su red clientelar, con individuos como su sobrino, el todopoderoso deán don Pedro de Salazar y Góngora, su secretario - don Bernardo Blázquez- o el canónigo don Juan Antonio de Victoria.

Prosiguiendo con este tipo de intervinientes, debemos añadir que lo previamente expuesto no impide que en las 
actas aparezcan, aunque de forma más suelta, un nutrido grupo de eclesiásticos diocesanos y regulares. Estos últimos aparecen en menor medida y son, entre otros, el canónigo don Diego Murillo - administrador capitular que interviene en cinco ocasiones entre 1686 y 1687, en una entre 1696 y 1697 y en otra más entre 1705 y 1706 debido a presentación de cuentas y a otros menesteres propios de su cargo-,${ }^{47}$ el canónigo don Jerónimo del Valle - albacea del cardenalobispo, e interviniente por dos veces para el primer año, por cuatro para el segundo y por otras cuatro para el último año- ${ }^{48}$ el canónigo don Francisco del Eraso - secretario capitular, que interviene en un total de cinco ocasiones repartidas irregularmente por el grueso de los tres años para la firma de los escritos capitulares $-{ }^{49}$ o el canónigo don Luis de Valenzuela - que desempeña un activo papel en la preparación de los fastos fúnebres de Salazar..$-^{50}$

Imperativos esencialmente económicos ${ }^{51}$ y caritativos ${ }^{52}$ son los motores que mueven al tercer estado a presentarse ante su cabildo catedralicio y, como vimos, su total sesenta y tres personas- se divide en trece para 16861687 , veintiuno para $1696-1697$ y veintinueve para 1705 1706. A pesar de que no son siempre las mismas personas las que se dirigen al cabildo a lo largo de estos tres años, sí se advierte en los documentos capitulares la constante presencia e intervención de todos los sectores económicos, si bien es cierto que con una preponderancia del sector terciario frente a los otros dos, y dentro de este, los que más habitualmente exponen sus problemas y/o formulan sus peticiones al cabildo, son abogados y administradores. Existe también, aunque en bastante inferior proporción, un inconexo grupo de inclasificables de los que poco o nada se puede decir más que su nombre y lo que le lleva a acudir al cabildo.

Analizando la evolución de las personas físicas del estamento nobiliario, notamos en las actas su prolongada y variada presencia a lo largo de los tres años testigos - quizá con una cierta concentración entre 1705 y 1706-, aunque no siempre de la mano de los mismos protagonistas salvo en casos puntuales. Si el total asciende a diez individuos: tres se encuadran dentro del primer año, uno para el segundo y seis para el tercero. Los nobles que más repetidamente intervienen en el cabildo son el duque de Arcos y el marqués de la Villa de Arias por motivos derivados de un pleito por el cobro de beneficios de una capilla en el caso del primero ${ }^{53}$ y con aspectos económicos derivados de la Guerra de Sucesión en el caso del segundo. ${ }^{54}$

También en las actas está presente, como pudimos comprobar, la realeza por las razones en otro apartado enunciadas. Son tres los miembros que de ella se tiene constancia en las actas, en las personas de Carlos II -que aparece tres veces; una dentro del primer año testigo y dos a lo largo del segundo-, ${ }^{55}$ Felipe $\mathrm{V}$-cuya intervención se

\footnotetext{
47 ACCO, t. 62, f. 198 r.

48 ACCO, t. 66, f. 352 v.

49 ACCO, t. 66, f. 355 r.

50 ACCO, t. 66, f. 355 r.

51 ACCO, t. 66, f. 239 v.

ACCO, t. 62, f. $96 \mathrm{v}$.

53 ACCO, t. 62, f. 153 v.

ACCO, t. 66, f. 320 r.

ACCO, t. 62 , f. 346 r.
}

registra de manera concentrada en el último de los tres años - y María Luisa Gabriela de Saboya - primera esposa del anterior monarca, que interviene en una ocasión, en idéntica fecha que su marido-.$^{56}$

Tocando la evolución cronológica de las personas jurídicas, el total de las instituciones eclesiásticas contenidas en la documentación asciende a veintidós -cuatro para $1686-1687$, doce para $1696-1697$ y seis para $1705-$ 1706- A la categoría de personas jurídicas, se adscriben en las actas organismos como los cabildos catedralicios de distintas partes de España - Toledo, Granada, Sevilla y Málaga especialmente-, la Santa Sede, hospitales como el de convalecientes de Málaga o el de niños expósitos de Córdoba y colegios como el de san Antonio. La única continuidad que se observa en las fuentes es la relativa a la presencia de los cabildos eclesiásticos y su correspondiente actuación, frente al resto de propiedades, solo aludidas puntual e intermitentemente.

Parándonos en el estudio de la continuidad de las personas jurídicas laicas, advertimos que esta es mínima y concentrada solo entre 1705 y 1706 en dos concejos municipales como los de la Puebla de Alférez ${ }^{57}$ y Córdoba ${ }^{58}$ por las razones también aludidas en el apartado de intervinientes. No obstante, su escasa presencia no es inconveniente para apreciar un ostensible repunte en el tercer año testigo, probablemente como reflejo de la paulatina secularización de la sociedad cordobesa dieciochesca. La evolución cronológica de las personas jurídicas del estamento nobiliario es nula por su inexistencia en los libros de actas, y la de la realeza es palpable de una forma leve pero continuada a lo largo de los tres años testigos en las personas de los monarcas de turno y por lo ya puesto sobre el tapete.

Realizados un seguimiento cronológico de los intervinientes físicos y jurídicos registrados en los libros de actas durante los tres años testigos, haremos lo propio con los asuntos que en ese tiempo el cabildo debe atender. Lo que nos desglosa la Tabla 5 es la evolución cronológica de los asuntos que, a lo largo del mencionado abanico cronológico, esta institución deberá dirimir durante la prelatura de Salazar. Sucesivamente comprobaremos la continuidad o no de estas temáticas viendo, en relación con lo en su momento expuesto en el apartado de asuntos, también si son o no solucionadas.

En primer lugar, no es que continúen las mismas casuísticas a lo largo del tiempo. Los temas son tan numerosos que su rastro se pierde en las actas a lo largo de los tres años. Esa circunstancia se explica por la mecánica capitular, rápida cuando de la resolución de conflictos se trata, pese a solo haber asumido tres años de la prelatura de Salazar. Lo que sí persisten son las categorías bajo las que se adscriben. Por ello podemos afirmar que son muchos y muy distintos los asuntos que se tratan y se reparten, salvo determinadas excepciones, de manera relativamente uniforme en el grueso de la documentación examinada.

Por este mismo motivo, una segunda reflexión es el mayor registro en las actas de algunos temas frente a otros. Aquí posiblemente está la explicación de porqué en

\footnotetext{
56 ACCO, t. 66, f. 266 r.

ACCO, t. 66, f. 243 r.

ACCO, t. 66, f. 255 v.
} 
TABLA 5

Evolución cronológica de los asuntos que dirime el cabildo catedralicio cordobés durante los tres años testigos

\begin{tabular}{|l|c|c|c|c|}
\hline \multicolumn{5}{|c|}{ TOTAL GLOBAL DE ASUNTOS: 283 (100 \%) } \\
\hline & $\begin{array}{c}\text { TOTALES } \\
\text { (No / \%) }\end{array}$ & $\begin{array}{c}1686-1687 \\
\text { (№ / \%) }\end{array}$ & $\begin{array}{c}1696-1697 \\
\text { (№ / \%) }\end{array}$ & $\begin{array}{c}1705-1706 \\
\text { (№ / \%) }\end{array}$ \\
\hline Personal del cabildo & $146 / 100$ & $43 / 29,45$ & $57 / 39,04$ & $46 / 31,51$ \\
\hline Asuntos económicos & $43 / 100$ & $14 / 32,56$ & $15 / 34,88$ & $14 / 32,56$ \\
\hline Relaciones cardenal-cabildo & $30 / 100$ & $10 / 33,33$ & $15 / 50$ & $5 / 16,67$ \\
\hline $\begin{array}{l}\text { Relaciones entre cabildos, } \\
\text { con la Santa Sede y Ultramar }\end{array}$ & $23 / 100$ & $9 / 39,13$ & $11 / 47,83$ & $3 / 13,04$ \\
\hline Relaciones Iglesia-Estado & $16 / 100$ & $5 / 31,25$ & $5 / 31,25$ & $6 / 37,5$ \\
\hline Asistencia a la sociedad & $14 / 100$ & $6 / 42,86$ & $4 / 28,57$ & $4 / 28,57$ \\
\hline Pleitos & $7 / 100$ & $2 / 28,57$ & $3 / 42,86$ & $2 / 28,57$ \\
\hline Asistencia al clero diocesano & $4 / 100$ & $1 / 25$ & $1 / 25$ & $2 / 50$ \\
\hline
\end{tabular}

Fuente: Elaboración propia.

los escritos capitulares aparecen más temáticas vinculadas con personal y economía del cabido frente a otros como pleitos o asistencia al clero diocesano, que casi brillan por su ausencia al serle externos.

Sin perder de vista estos planteamientos, analizaremos la evolución cronológica de cada categoría de asuntos que el cabildo solventa. Empezaremos por la correspondiente a su personal. Su presencia en los documentos es de ciento cuarenta y seis asuntos, repartidos en cuarenta y tres para el primer año, cincuenta y siete para el segundo y cuarenta y seis para el tercero. Pese a su relativamente homogénea prolongación en el tiempo, es el segundo año el más dinámico en este aspecto por ser cuando más relevos de vacantes se producen y el cardenal Salazar interviene más activamente.

Las cuestiones de personal oscilan a lo largo del tiempo. Por lo general van de menos a más, quizá parejas al ritmo de la labor de Salazar. Tardan varios meses como máximo en finiquitarse y en las fuentes no consta que un asunto se prolongue indefinidamente a lo largo del período estudiado.

El segundo gran bloque de asuntos presente en la documentación es el económico. Son cuarenta y tres los asuntos de esta naturaleza: catorce para 1686-1687, quince para 1696-1697 y catorce para 1705-1706.

Como decíamos, no se aprecia rastro de asuntos concretos perdurables en la documentación en el grueso de los tres años, puesto que es muy raro que se alarguen imprecisamente -el proceder general del cabildo, en sintonía con el de sus homólogos repartidos por la geografía española, ${ }^{59}$ es resolver el tema cuanto antes en la medida de los posible-, pero sí que hay continuidad en las acciones que el cabildo con su obispo a la cabeza efectúan en este

\footnotetext{
59 García-Cuevas Ventura 1984: 99.
}

terreno. En las actas, la actividad económica que más se refleja es la de la presentación de cuentas ya sea de parroquias y fábricas, ${ }^{60}$ como de obras pías, ${ }^{61}$ propiedades del obispado, ${ }^{62} \mathrm{y}$, especialmente, presupuestos anuales $\mathrm{y}$ sueldo de los capitulares. ${ }^{63}$

Todas ellas se ponen sobre la mesa capitular, ante el prelado y sus subordinados, siendo ellos los que deciden, tras una prudente espera, si deben aprobarse o no estos presupuestos. Como se detecta en las actas, no es que lo anterior sea una peculiaridad de Salazar, dado que también encontramos esta casuística en otros cabildos catedralicios españoles como lo propio de las funciones de todo obispo, si bien es verdad, que la tensión que en Córdoba se deriva de este hecho no se da en los otros organismos.

\section{CONCLUSIÓN}

Concluiremos parcialmente este estudio recogiendo sucintamente las observaciones que se han ido desarrollando en las páginas anteriores y que por el avance de nuestra investigación sobre el cardenal Salazar, serán modificadas, al ser más lo que hay todavía por descubrir sobre este tema que lo ya dado a conocer.

El primer postulado a recordar, es que, como ya hemos indicado al comienzo de este artículo, este trabajo se plantea como un estudio entre lo social y lo institucional, con una clara vocación nacional aunque se haya visto a través del prisma local -el de Córdoba, buen paradigma, porque era un obispado interesante y un cabildo poderoso-.

Como segunda reflexión, sostenemos un condicionamiento de la actividad episcopal del cardenal Salazar tanto por la sociedad cordobesa de finales del Seiscientos y principios del Setecientos, como por su propia persona. La misma importancia que la referida sociedad concede a sus problemas es la que el purpurado concede a las disposiciones tridentinas, con todo lo que ello implica en la capacidad de gestión del cabildo en los problemas de la ciudad.

También hemos podido constatar que el cabildo catedral cordobés es una institución que se comporta y funciona como tal, pese a las tiranteces que mantiene con su obispo en época de Salazar. También a lo largo de la realización de este trabajo, hemos podido empezar a intuir y desentrañar ciertas claves del aludido organismo a través de la sociedad que pasa por él y lo que le preocupa, pudiendo demostrar su cercanía con la sociedad cordobesa del momento y sus prolíficas relaciones con las restantes instituciones eclesiásticas y con otras tantas de naturaleza civil.

Finalmente, debemos establecer una perfecta correlación de la tónica imperante en el cabildo catedralicio cordobés con la que podemos encontrar en los restantes cabildos diocesanos españoles pese a las en ocasiones extremas tiranteces entre Salazar y sus capitulares.

Desde la Historia Social contribuiremos también así a seguir ensanchando el conocimiento de la Iglesia española del Antiguo Régimen como institución social y de poder. 


\section{FUENTES PRIMARIAS}

1686-1687

Archivo Capitular de la Catedral de Córdoba, en lo sucesivo ACCO, t. 62: ff. 96 v; 103 v; 153 v; 160 v; 179 v; 198 r; 346 r.; 367 r; 368 ry 405 r.

1696-1697

ACCO, t. 64: ff. 234 r; 405 r; 416 v; 425 v; 431 r; 438 v y 461 v.

1705-1706

ACCO, t. 66: ff. 239 v; 243 r; 255 v; 266 r; 294 v; 315 v; 320 r; 330 r; 346 r; 352 v; 355 r y 377 v.

\section{BIBLIOGRAFÍA}

Álvarez Santaló, L. C. 2010. «Vivir como un cura: algunas percepciones cuantitativas respecto al imaginario social sobre el clero en el siglo XVII», en F. Aranda Pérez (coord.), Sociedad y élites eclesiásticas en la España moderna: 101-157. Cuenca: Ediciones de la Universidad de Castilla - La Mancha.

Aranda Doncel, J. 1984. La época moderna (1517-1808) 3. Córdoba: Caja de Ahorros del Monte de Piedad de Córdoba - CSIC.

Barrio Gozalo, M. 2010. El clero en la España moderna. Córdoba: Monte de Piedad y Caja de Ahorros de Córdoba.

Cuenca Toribio, J. M. 2005. Estudios sobre el catolicismo español contemporáneo 4. Córdoba: Servicio de Publicaciones de la Universidad de Córdoba.

Domínguez Ortiz, A. 1978. «El Barroco», en A. Domínguez Ortiz, J. M. Pita Andrade y J. Fradejas, El Barroco: 212-220. Madrid: Ministerio de Cultura.

Domínguez Ortiz, A. 1985. Las clases privilegiadas del Antiguo Régimen: el clero. Madrid: Istmo.

García-Cuevas Ventura, J. 1996. El cabildo catedralicio cordobés desde la revolución a la Restauración (1788-1882). Córdoba: Servicio de Publicaciones de la Universidad de Córdoba.
Gómez Navarro, S. 2004. «El cardenal Salazar y la política proborbónica de su tiempo», en S. Gómez Navarro (coord.), Estudios de Historia Iberoamericana II. Reunión Anual de la Society for Spanish and Portuguese Historical Studies (SSPHS). Córdoba: Servicio de Publicaciones de la Universidad / Cajasur.

Gómez Navarro, S. 2006. «Empezando a pastorear: la misión del cardenal Salazar en la Salamanca de 1682», en J. M. de Bernardo Ares (coord.), La Sucesión en la Monarquía Hispánica, 16651725. Córdoba: Servicio de Publicaciones de la Universidad / Cajasur.

Jiménez Martínez, M. J. 2007. El epistolario como fuente auxiliar para el conocimiento del cabildo catedralicio de Málaga en el reinado de Fernando VI (1746-1759).Tesis doctoral, Universidad de Málaga

Morgado García, A. 2000. Ser clérigo en la España del Antiguo Régimen. Cádiz: Servicio de Publicaciones de la Universidad de Cádiz.

Peña Díaz, M. 2014. "Mirando al cielo. Clima y religiosidad en los siglos XIV y XVII», en M. Peña Díaz (coord.), Vivir en el Siglo de Oro. Andalucía en la Historia 44 (1-2). Centro de Estudios Andaluces.

Pons Alós, V. y Cárcel Ortí, M. 2013. «Dignidades y canónigos de la catedral de Valencia en el siglo XVIII», en E. Callado Estela (coord.), La catedral ilustrada. Iglesia, sociedad y cultura en la Valencia del siglo XVIII. Valencia: Instituciò Alfons el Magnànim - Diputación Valencia.

Reder Gadow, M. 2000. «Felipe II, Trento y la diócesis de Málaga». Hispania Sacra 52 (105): 338-402. https://doi.org/10.3989/hs.2000.v52.i106.561

Soria Mesa, E. 2000. El cambio inmóvil: transformaciones y permanencias de una élite de poder (Córdoba, ss. XVI-XIX). Córdoba: Ayuntamiento de Córdoba.

Vázquez Lesmes, R. 1987. Córdoba y su cabildo catedralicio en la Modernidad. Córdoba: Monte de Piedad y Caja de Ahorros de Córdoba. 